\title{
Considerações Sobre o Tratamento da Esclerose Múltipla
}

\author{
Nilton Amorim de Souza* \\ Enedina Maria Lobato de Oliveira**
}

\section{RESUMO}

A esclerose múltipla é uma doença do sistema nervoso central de etiologia inflamatória, presumidamente auto-imune e caracterizada pela destruição da mielina. Alguns aspectos do seu tratamento são abordados.

\section{UNITERMOS}

Esclerose múltipla, interferon-beta, imunossupressores, plasmaferese, imunoglobulina.
Pós-graduando em Neurologia, Setor de Doenças Neuromusculares da Unifesp EPM.

* * Pós-graduanda em Neurologia, Setor de Doenças Neuromusculares da Unifesp EPM. Laboratory of Molecular Immunology, Center for Neurologic Diseases, Brigham and Women's Hospital - Harvard Medical School.

\section{INTRODUÇÃO}

Uma vez estabelecido o diagnóstico de esclerose múltipla (EM), algumas considerações são necessárias, tais como: possibilidades terapêuticas e a subseqüente evolução clínica, ambas diretamente relacionadas, atualmente, às novas drogas disponíveis.

Embora não completamente definidos, existem fortes evidências de mecanismos imunológicos na patogênese da doença, o que tem motivado, ao longo dos anos, o uso de drogas imunossupressoras e imunomoduladoras de ação inespecífica, assim como a pesquisa exaustiva de tratamentos mais específicos. Tais tratamentos têm como principal objetivo controlar ou modular a população-alvo de células $\mathrm{T}$ auto-reativas, que parece desempenhar um papel importante na fisiopatologia do processo inflamatório-desmielinizante.

A idéia de que a EM é uma doença sem tratamento está mudando progressivamente, com o aparecimento de drogas que influenciam seu curso clínico. Apesar do surgimento de uma assim chamada terapia de "primeira linha", que nos dá algum alento e conforto, precisamos ter em mente que o tratamento da EM envolve muito mais. Ainda, compete-nos melhorar a qualidade de vida diária, interferindo na incapacidade adquirida, implementando novas técnicas de reabilitação e desenvolvendo drogas ou técnicas que restaurem ou induzam a remielinização de áreas previamente lesadas.

Apesar de ainda estarmos no início da jornada em busca de tratamentos curativos para as doenças auto-imunes, em relação à EM, podemos nos permitir um certo otimismo.

\section{TRATAMENTO DAS EXACERBAÇÕES (SURTOS) Glicocorticóides}

Os glicocorticóides constituem a primeira linha de tratamento para os surtos de esclerose múltipla. Os mecanismos de ação dos glicocorticóides são diversos. Inibem a proliferação de linfócitos $\mathrm{T}$, suprimem a produção e a ativação de citoquinas, restauram a barreira hematoencefálica e alteram a transmissão axonal ${ }^{1,2}$. Foi demonstrado que os glicocorticóides reduzem a expressão de moléculas de adesão na superfície das células mononucleares 
do sangue e LCR de pacientes com $\mathrm{EM}^{3}$. Os primeiros ensaios clínicos em EM utilizaram não a administração direta de corticóides, mas sim do hormônio adrenocorticotrófico (ACTH). Um estudo multicêntrico comparou os resultados do tratamento dos surtos de EM entre o grupo tratado com ACTH e o grupo-placebo ${ }^{4}$, utilizando a escala de Kurtzke para avaliação funcional e do grau de incapacidades neurológicas dos pacientes ${ }^{5}$. A dose utilizada foi de 40 UI IM, duas vezes ao dia, durante 7 dias; 20 UI, duas vezes ao dia, durante 4 dias e de 20 UI, uma vez ao dia, durante 3 dias. O tratamento com ACTH resultou em melhor recuperação.

A metilprednisolona intravenosa (MPIV) constitui, atualmente, a droga de primeira escolha para o tratamento dos surtos, com resultados melhores e mais consistentes em relação ao $\mathrm{ACTH}^{6,7}$. Os resultados do Optic Neuritis Treatment Trial ${ }^{8}$ têm sido extrapolados para todos os surtos de EM. Esse estudo demonstrou que um regime de MPIV (1.000 mg/dia, durante 3 dias), seguido de prednisona ( $1 \mathrm{mg} / \mathrm{kg} / \mathrm{dia}$, durante $11 \mathrm{dias})$, acelerou a recuperação do déficit visual. Entretanto, após 6 semanas não houve diferença significativa na acuidade visual entre os grupos MPIV e placebo. Porém, os resultados mais importantes foram a constatação de que, durante a fase aguda, o tratamento com MPIV (Solumedrol ${ }^{\circledR}$ ) reduz o risco de desenvolvimento de EM durante um período de 2 anos seguidos à terapia ${ }^{9}$. O risco de ser diagnosticada EM clinicamente definida, durante esse período, foi de $7,5 \%$ no grupo MPIV, $14,7 \%$ no grupoprednisona e $16,7 \%$ no grupo-placebo. Esse estudo deve ser interpretado sabendo-se que não foi desenhado para avaliar a taxa de recorrência de surtos na EM. Um outro estudo, utilizando ressonância nuclear magnética encefálica (RNM), contrastada com gadolínio, sugeriu que o tratamento com MPIV, seguido por um curso rápido de prednisona oral, reduz o número de lesões novas durante um seguimento de 6 meses $(1,5$ novas lesões/scan com tratamento vs 2,25 lesões/scan sem tratamento $)^{10}$. A metilprednisolona oral tem sido utilizada por alguns clínicos nas exacerbações de EM, devido à facilidade e à redução dos custos de administração. Os dados quanto ao benefício equivalente não foram suficientemente persuasivos ${ }^{11,12,13}$. Até este momento, a despeito de alguns resultados positivos nesses estudos, a conduta recomendada é a administração intravenosa da metilprednisolona para o tratamento dos surtos de EM, na dose de $1 \mathrm{~g} / \mathrm{dia}$, durante três a cinco dias. O tratamento crônico com corticóides (prednisona oral) está contra-indicado, uma vez que não altera a progressão neurológica ou o número de surtos, podendo contribuir para a piora do quadro ${ }^{14}$. Alguns efeitos colaterais dos glicocorticóides são: hipertensão arterial, intolerância à glicose, obesidade, aparência cushingóide, osteoporose, necrose óssea asséptica, catarata, irritabilidade, alterações do humor e insônia.

\section{PREVENÇÃO DOS SURTOS E DA PROGRESSÃO}

\section{Interferons}

As tentativas iniciais no uso dos interferons em EM foram baseadas em suas propriedades antivirais e na crença de uma infecção viral persistente como causa da doença.

Os primeiros ensaios terapêuticos envolveram administração sistêmica e intratecal de ambos os interferons, alfa e beta. O número de pacientes nesses estudos foi pequeno e os resultados, conflitantes ${ }^{15}$. A despeito dos avanços clínicos, os mecanismos de ação do interferon (IFN) na EM ainda não estão plenamente esclarecidos. Sabe-se que o IFN- $\beta$ inibe a produção de IFN- $\gamma$, este último, uma citoquina pró-inflamatória com múltiplas funções, incluindo a ativação de macrófagos e linfócitos e o aumento da expressão de moléculas de adesão na superfície das células endoteliais, responsáveis pela migração de células T para o SNC. Acredita-se que o IFN- $\beta$ tenha efeito inibitório sobre a proliferação linfocitária e que a apresentação antigênica desvie a produção de citoquinas na direção de um perfil antiinflamatório e reduza a migração das células $\mathrm{T}^{16}$. No modelo murino de encefalite auto-imune experimental (EAE), uma condição inflamatória em camundongos que se assemelha à EM, o IFN- $\beta$ mostrou-se eficaz, reduzindo a progressão da doença ${ }^{17}$.

\section{Interferon- $\boldsymbol{\beta} \mathbf{1 b}$}

Em 1993, dois grandes estudos demonstraram que o interferon- $\beta 1 \mathrm{~b}$ (IFN- $\beta 1 \mathrm{~b}$ ), uma forma recombinante $\mathrm{e}$ modificada do interferon- $\beta$ humano, reduz a frequiência de surtos na EM forma surto-remissiva ${ }^{18,19}$. Também foi constatada a redução no número e freqüência de lesões encefálicas na RNM. O seguimento de quatro a cinco anos dos pacientes recebendo IFN- $\beta 1$ b mostrou redução na taxa de surtos em um terço dos pacientes, com maior número de pacientes permanecendo livres de surtos por tempo mais prolongado quando comparados com o grupo-placebo. A RNM encefálica seriada revelou aumento do número de lesões no grupo-placebo, porém não significativamente no grupo tratado ${ }^{20,21}$.

Estudos posteriores comprovaram que o IFN- $\beta 1 \mathrm{~b}$, além de diminuir a freqüência e a gravidade dos surtos 
de EM e de reduzir a atividade da doença na RNM encefálica, também altera a progressão da incapacidade neurológica nas formas surto-remissivas $18,19,20,21$.

Recentemente, foi demonstrado o efeito benéfico do IFN- $\beta 1$ b sobre a progressão da incapacidade adquirida nas formas secundariamente progressivas de $\mathrm{EM}^{22}$. Nesse estudo, foram incluídos pacientes com EM, EDSS (Expanded Disability Status Scale) variando de 3,0 a 6,5 e com doença em atividade por dois anos. O tratamento consistiu na administração de oito milhões de UI de IFN- $\beta$ 1b ou placebo, via subcutânea, em dias alternados, sendo os resultados analisados após dois anos de uso. O tempo decorrido necessário para confirmar deterioração neurológica foi significativamente maior nos pacientes tratados em relação ao placebo. Houve redução de, aproximadamente, $30 \%$ na taxa de surtos e redução da necessidade de uso de corticóides e de hospitalizações. Do ponto de vista de neuroimagem, houve redução significativa no volume total de lesões encefálicas nas sequiências ponderadas em T2 à RNM e menor aparecimento de lesões novas.

Em virtude desses resultados, preconiza-se atualmente o uso de IFN- $\beta$ 1b nas formas secundariamente progressivas de EM, na dose de 8 milhões UI, via subcutânea, em dias alternados. A medicação em geral é segura, com efeitos colaterais transitórios do tipo flulike (febre, mialgias, calafrios, cefaléia e fadiga), reações cutâneas locais, elevação das enzimas hepáticas ou anormalidades na contagem de leucócitos. Em geral, tais efeitos colaterais podem durar até três meses após o início do tratamento. O IFN- $\beta$ não deve ser usado na gestação.

\section{Interferon- $\beta$ la}

$O$ interferon- $\beta$ 1a difere do interferon- $\beta$ 1b pela substituição de um único resíduo de aminoácido e por ser glicosilado ${ }^{23}$.

Diversos estudos foram realizados utilizando-se o IFN- $\beta$ 1a em doses variadas, cuja eficácia clínica ficou demonstrada pela redução da freqüência e gravidade dos surtos. Os estudos iniciais com doses de 6 milhões de UI semanais detectaram redução da taxa de surtos em, aproximadamente, $30 \%{ }^{24}$. Novos estudos foram realizados utilizando-se doses progressivamente maiores, com 3 e 9 M milhões UI, 3 vezes por semana. O estudo PRISMS utilizou doses de 6 MUI $(22 \mu \mathrm{g})$ ou 12 MUI (44 $\mu \mathrm{g}$ ) ou placebo, por via subcutânea, 3 vezes por semana, durante $2 \operatorname{anos}^{25}$. O número de surtos foi significativamente inferior no primeiro e no segundo ano com as duas doses de interferon em relação ao grupoplacebo (redução de $37 \%$ após 1 ano e $32 \%$ após 2 anos com 12 MUI e de $33 \%$ e de $29 \%$ com 6 MUI, respectivamente). O intervalo para aparecimento do primeiro surto, após o início da medicação, foi mais prolongado em relação ao placebo e houve redução dos surtos moderados e graves, assim como do número médio de lesões ativas verificadas à RNM encefálica. Atualmente, recomendam-se doses maiores de IFN- $\beta$ 1a para as formas surto-remissivas de EM.

\section{Acetato de glatiramer}

Trata-se de uma mistura de peptídeos randomizados de quatro aminoácidos, L-alanina, L-lisina, L-tirosina e L-ácido glutâmico ${ }^{23}$. O mecanismo de ação exato é desconhecido. Parece haver reação cruzada com a proteína básica de mielina (MBP). O tratamento prévio com acetato de glatiramer diminui a incidência e a gravidade da encefalite auto-imune experimental. Os mecanismos postulados incluem dessensibilização, aumento da produção de células T supressoras antígeno-específicas, inibição das células T efetoras MBP-específicas e competição com o sítio de ligação do antígeno da classe II do MHC (complexo maior de histocompatibilidade) $)^{26,27}$.

$\mathrm{O}$ acetato de glatiramer foi utilizado em um estudo de 252 pacientes com EM forma surto-remissiva (EDSS entre 0,0 a 5,0), divididos em grupos placebo e tratamento. A dose foi de $20 \mathrm{mg}$ diariamente, via subcutânea. Houve redução de $30 \%$ na taxa anual de surtos no grupo tratado em relação ao placebo ${ }^{26,27}$. Uma extensão do estudo demonstra que o efeito favorável sobre os surtos persiste ${ }^{28}$. A droga é bem tolerada, com leves sintomas sistêmicos consistindo em flushing, sensação de opressão torácica, palpitações, ansiedade e dispnéia, ocorrendo em $15 \%$ dos pacientes. Não foram observadas alterações hematológicas ou bioquímicas e as reações no local da injeção são leves. Não deve ser usado na gestação.

\section{Imunossupressores}

\section{Ciclofosfamida}

A ciclofosfamida é um agente alquilante que interfere na síntese e função do DNA. Tem a capacidade de reverter o curso da EAE quando administrada antes ou $\operatorname{logo}$ após o início dos sintomas ${ }^{29}$. Diversos estudos foram realizados em EM formas surto-remissivas e progressivas, sem evidência clara de sua eficácia terapêutica ${ }^{30,31,32}$. Existem protocolos atuais que utilizam a ciclofosfamida em pulsos mensais nas formas progressivas de EM. A dose preconizada é de 600 a $1.200 \mathrm{mg} / \mathrm{m}^{2}$ de superfície corpórea IV, administrados mensalmente, sendo que alguns associam dose prévia de metil- 
prednisolona $\mathrm{IV}^{33}$. A ciclofosfamida parecer alterar o perfil da resposta imunológica na EM, reduzindo a produção de interleucinas amplificadoras da resposta inflamatória, especialmente interleucina $12^{33}$. A toxicidade da ciclofosfamida, a longo prazo, limita sua utilização crônica, incluindo-se a ocorrência de neoplasia de bexiga e leucemias, além de efeitos colaterais secundários: cistite hemorrágica, amenorréia, oligospermia, alopecia e susceptibilidade a infecções ${ }^{34}$.

\section{Mitoxantrona}

Trata-se de um agente antineoplásico com potente efeito imunomodulador, suprimindo a imunidade mediada pelas células $\mathrm{T}$ e $\mathrm{B}$.

Os estudos realizados, até o momento, têm demonstrado supressão sobre a atividade da doença, tanto nas formas surto-remissivas quanto secundariamente progressivas, medidas em parâmetros clínicos e à RNM encefálica ${ }^{35,36,37}$. Observou-se redução na taxa de surtos e na progressão da incapacidade neurológica, medida pelo EDSS, na ausência de efeitos tóxicos graves em relação a outros imunossupressores. A cardiotoxicidade é o efeito colateral mais grave, podendo ser dose-dependente e cumulativo, o que limita o uso crônico da medicação. Novos estudos estão em andamento no sentido de obter conclusões mais precisas.

\section{Outros agentes}

\section{I munoglobulina}

Constitui-se em um pool de IgG humana com diversas propriedades imunorreguladoras.

Os estudos sobre o uso de imunoglobulina intravenosa (IGIV) nas formas surto-remissivas de EM têm demonstrado resultados favoráveis. IGIV na dose de 150 a $200 \mathrm{mg} / \mathrm{kg}$ em dose única mensal, durante 2 anos, mostrou-se eficaz em reduzir a taxa de surtos (em torno de 50\%) e a gravidade dos mesmos, porém com discreta interferência sobre o EDSS ${ }^{38}$. Estudos utilizando doses maiores têm apresentado resultados controversos quanto à redução das lesões encefálicas ativas à RNM ou à evolução clínica ${ }^{39,40}$.

Até o momento, não há consenso para o uso de IGIV de forma rotineira na prática clínica com base nos estudos disponíveis.

\section{Plasmaferese}

A plasmaferese $(\mathrm{PF})$ é um recurso terapêutico já reconhecido e eficaz em algumas doenças neurológicas de caráter imunológico, como a síndrome de Guillain e Barré e a miastenia gravis. No caso da EM, vários estudos têm sido realizados cujos resultados parecem apontar para um efeito benéfico sobre a doença.

Vamvakas e Weinshenker ${ }^{41}$, após a realização de metaanálise sobre o uso da PF nas formas progressivas de EM, concluíram haver diminuição na incapacidade adquirida. Entretanto, a análise foi prejudicada devido à interferência de diversos fatores, como o uso prévio de imunossupressores nos casos e controles, diferenças entre os diversos protocolos de PF e inclusão de formas clínicas primárias e, secundariamente, progressivas na maioria dos estudos.

Até o momento, não há consenso sobre o uso da PF nas formas progressivas de EM.

$\mathrm{Na}$ forma surto-remissiva, a PF tem sido usada para tratamento dos surtos corticóide-resistentes ${ }^{42,43}$. Rodriguez et al. ${ }^{43}$ demonstraram resposta dramaticamente favorável em surtos de EM não responsivos a corticosteróides, tratados com seis a nove sessões de PF em dias alternados, sem uso concomitante de imunossupressores. Estudos randomizados duplo-cegos encontram-se em andamento para confirmar os resultados.

\section{CONCLUSÃO}

Ainda distantes de uma abordagem curativa, os avanços recentes permitem oferecer tratamento relativamente efetivo para os portadores de esclerose múltipla. Pulsoterapia com metilprednisolona intravenosa é o procedimento estabelecido para tratamento das exacerbações, seguida ou não de um período curto de prednisona oral em doses decrescentes. A forma surto-remissiva, em nosso meio, tem sido tratada preferencialmente com interferon, embora em outros centros a droga de primeira escolha seja o acetato de glatiramer. Não há comparação formal entre os interferons e o acetato de glatiramer para conclusões mais precisas sobre suas eficácias relativas. As formas secundariamente progressivas parecem beneficiar-se do tratamento com INF- $\beta 1 \mathrm{~b}$, enquanto que os quadros primariamente progressivos devem ser tratados com ciclofosfamida.

Sem dúvida, os estudos laboratoriais e os modelos experimentais contribuem para que as fronteiras terapêuticas se ampliem. Novos agentes que visam controlar fases específicas da resposta imunológica, tais como expressão de moléculas de adesão, de moléculas coestimulatórias, adesão e migração linfocitária e produção de citoquinas, estão em diferentes fases de investigação clinicolaboratorial. No entanto, as perspectivas a longo prazo são alentadoras. 


\section{SUMMARY}

Treatment of multiple sclerosis: general aspects

Multiple sclerosis is a chronic inflamatory immuno-mediated demyelinating disease of the central nervous system, and currently one of the most common disabling neurologic disease of young people. We disccuss some recent developments in its therapy.

\section{KEYWORDS}

Multiple sclerosis, interferon-beta, imunossupressors, plasmaferese, immunoglobulin.

\section{Referências}

1. Pachner AR. Immunosupressive therapy. Targeting the autoantigen in neurologic disease. Adv Neuroimmunology, 4:310, 1997.

2. Weinstock-Guttman B, Cohen JA. Emerging therapies for multiple sclerosis. Neurologist, 2:342-55, 1996.

3. Elovaara I, Lalla M, Spare E, Lehtimaki T, Dastidar P. Methylprednisolone reduces adhesion molecules in blood and cerebrospinal fluid in patients with MS. Neurology, 51:17031708, 1998.

4. Rose AS, Namerow NS, Kuzzma JW et al. Cooperative study in the evaluation of therapy in multiple sclerosis. Neurology, 20:1-59, 1970

5. Kurtzke JF. Rating neurologic impairment in multiple sclerosis: the Extended Disability Status Scale. Neurology, 33:1444-1452, 1983.

6. Milligan NM, Newcombe R, Compston DAS. A double-blind, controlled trial of high dose methylprednisolone in patients with multiple sclerosis. I: clinical effects. J Neurol Neurosurg Psychiatry, 50:511-16, 1987

7. Barnes MP, Bateman DE, Cleland PG et al. Intravenous methylprednisolone for multiple sclerosis relapse. J Neurol Neurosurg Psychiatry, 48:157-9, 1985.

8. Beck RW, Cleary PA, Anderson MM et al. A randomized controlled trial of corticosteroids in the treatment of acute optic neuritis. N Engl J Med, 326:581-8, 1992.

9. Beck RW, Cleary PA, Trobe JD et al. The effect of corticosteroids for acute optic neuritis on the subsequent development of multiple sclerosis. N Engl J Med, 329:17649, 1993

10. Smith ME, Stone LA, Alberts PS et al. Clinical worsening in multiple sclerosis is associated with increased frequency and area of gadopentate dimeglumine-enhancing magnetic resonance imaging lesions. Ann Neurol, 33:480-6, 1993.

11. Barnes D, Hughes RA, Morris RW et al. Randomized trial of oral and intravenous methylprednisolone in acute relapses of multiple sclerosis. Lancet, 349:902-6, 1997.

12. Alam SM, Kyriakides T, Lawden M et al. Methylprednisolone in multiple sclerosis. A comparision of oral and intravenous therapy at equivalent high dose. J Neurol Neurosurg Psychiatry, 56:1219-20, 1993.

13. Sellebjerg F, Frederiksen JL, Nielsen PM, Olesen J. Doubleblind, randomised, placebo-controlled study of oral, high-dose methylprednisolone in attacks of MS. Neurology, 51:529-534, 1998.

14. Miller JHD, Vas CJ, Noronha MJ, Liversedge LA, Rawson MD. Long-term treatment of multiple sclerosis with corticotrophin. Lancet, 2:429-31, 1967.

15. Durelli L, Bongioanni MR, Cavallo $\mathrm{R}$ et al. Chronic systemic high-dose recombinant interferon alpha-2a reduces exacerbation rate, MRI signs of disease activity, and lynfocyte interferon gamma production in relapsing-remitting multiple sclerosis. Neurology, 44:406-13, 1994.
16. Yong VW, Chabot S, Stuve O, Williams G. Interferon beta in the treatment of multiple sclerosis. Neurology, 51:682-689, 1998.

17. Yu M, Nishiyama A, Trapp BD, Tuohy VK. Interferon-b inhibits progression of relapsing-remitting experimental autoimmune encephalomyelitis. J Neuroimmunol, 64:91-100, 1996.

18. IFN- $\beta$ Multiple Sclerosis Study Group. Interferon beta $1-b$ is effective in relapsing-remitting multiple sclerosis. I. Clinical results of a multicenter, randomised, double-blind, placebocontrolled trial. Neurology, 43:655-661,1993.

19. Paty DW, Li DKB, UBC MS/MRI Study Group, IFN- $\beta$ Multiple Sclerosis Study Group. Interferon beta- $1 \mathrm{~b}$ is effective in relapsing-remitting multiple sclerosis. II. MRI analyses results of a multicenter, randomised, double-blind, placebocontrolled trial. Neurology, 43:662-667, 1993.

20. Lublin FD, Whitaker JN, Eidelman BH, Miller AE, Arnason $B G W$, Burks JS. Management of patients receiving interferon beta-1b for multiple sclerosis: report of a consensus conference. Neurology, 46:12-18, 1996.

21. IFN- $\beta$ Multiple Sclerosis Study Group, University of British Columbia MS/MRI Analysis Group. Interferon beta-1 a in the treatment of multiple sclerosis: final outcome of randomised controlled trial. Neurology, 45:1277-1285, 1995.

22. European Study Group on Interferon Beta-1b in Secondary Progressive MS. Placebo-controlled multicentre randomised trial of interferon beta- $1 \mathrm{~b}$ in the treatment of secondary progressive multiple sclerosis. Lancet, 352:1491-1497, 1998.

23. Bashir K, Whitaker JN. Current immunotherapy in multiple sclerosis. Immunol. Cell Biol, 76:55-64, 1998.

24. Jacobs L, Cookfair D, Rudick $R$ et al. Intramuscular interferon beta-1 a for disease progression in relapsing-remitting multiple sclerosis. Ann Neurol, 39:194-285, 1996.

25. PRISMS (Prevention of Relapses and Disability by Interferon Beta-1 a Subcutaneously in Multiple Sclerosis) Study Group. Randomised double-blind placebo-controlled study of interferon b-1 a in relapsing-remitting multiple sclerosis. The Lancet, 352:1498-1504, 1998.

26. Johnson KP, Brooks BR, Cohen JA et al. Copolymer-1 reduces relapse rate and improves disability in relapsing-remitting multiple sclerosis: results of a phase III multicenter, doubleblind, placebo-controlled trial. The Copolymer-1 Multiple Sclerosis Study Group. Neurology, 45:1268-76, 1995.

27. Bornstein MB, Miller A, Slagle E et al. A pilot trial of Cop-1 in exacerbating-remitting multiple sclerosis. N Engl J Med, 317:408-14, 1987

28. Johnson KM, Brooks BR, Cohen JA et al. Extended use of glatiramer acetate (Copaxone) is well tolerated and maintains its clinical effect on multiple sclerosis relapse rate and degree of disability. Neurology, 50:701-708, 1998.

29. Becker CC, Gidal BE, Fleming JO. Immunotherapy in multiple sclerosis, part 2. Am J Heath-Syst Pharm, 52:2105-20, 1995.

30. Hauser SL, Dawson DM, Lerich JR et al. Intensive immunosupression in progressive multiple sclerosis: a randomised three-arm study of high dose intravenous cyclophosphamide, plasma exchange and ACTH. N Engl J Med, 308:173-80, 1983.

31. The Canadian Cooperative Multiple Sclerosis Study Group The Canadian Cooperative trial of cyclophosphamide and plasma exchange in progressive multiple sclerosis. Lancet, 337:442-6, 1991

32. Weiner HL, Mackin GA, Orav EJ et al. Intermittent cyclophosphamide pulse therapy in progressive multiple sclerosis: final report of the Northeast Cooperative Multiple Sclerosis Treatment Group. Neurology, 43:910-18, 1993.

33. Takashima H, Smith DR, Fukaura H, Khoury S, Hafler DA, Weiner HL. Pulse cyclophosphamide plus methylprednisolone induces myelin-antigen-specific IL-4-secreting T cells in multiple sclerosis patients. Clin Immunol Immunopathol, 88:28-34, 1998. 
34. Schluep M, Bogousslavsky J. Emerging treatments in multiple sclerosis. Eur Neurol, 38:216-221, 1997.

35. Edan G, Miller DH, Clanet $\mathrm{M}$ et al. Therapeutic effect of mithoxantrone combined with methylprednisolone in multiple sclerosis: a randomised multicentre study of active disease using MRI and clinical criteria. J Neurol Neurosurg Psychiatry, 62:112-118, 1997.

36. Millefiorini E, Gasperini C, Pozzili C et al. Randomised placebo-controlled trial of mitoxantrone in relapsing-remitting multiple sclerosis: 24 month clinical and MRI outcome. J Neurol, 244:153-534, 1997.

37. Hartung HP, Gonsette R and the MIMS Study Group. Mitoxantrone in progressive multiple sclerosis: a placebocontrolled, randomised, observer-blind, european phase III multicentre study: clinical results. Multiple Sclerosis, 4:325, 1998.

38. Fazekas F, Deisenhammer F, Strasser-Fuchs S et al. Randomised placebo-controlled trial of monthly intravenous immunoglobulin therapy in relapsing-remitting multiple sclerosis. The Lancet, 349:589-593, 1997.

39. Sorensen PS, Wanscher B, Jensen CV et al. Intravenous immunoglobulin $\mathrm{G}$ reduces MRI activity in relapsing multiple sclerosis. Neurology, 50:1273-1281, 1998.
40. Achiron A, Gabbay U, Gilad R et al. Intravenous immunoglobulin treatment in multiple sclerosis. Neurology, 50:398-402, 1998.

41. Vamvakas E, Weinshenker B. Meta-analysis of clinical studies of the efficacy of plasma exchange in the treatment of chronic progressive multiple sclerosis. J Clin Apheresis, 10:163-170, 1995.

42. Weiner HL, Dau PC, Khatri $\mathrm{O}$ et al. Double-blind study og true vs. Sham plasma exchange in patients treated with immunosupression for acute attacks of multiple sclerosis. Neurology, 39:1143-1149, 1989.

43. Rodriguez M, Karnes J, Bartleson JD, Pineda AA. Plasmapheresis in acute episodes of fulminat CNS inflamatory demyelination. Neurology, 43:1100-1104, 1993.

\section{Endereço para correspondência:}

Dr. Nilton A. Souza

Unifesp-EPM - Disciplina de Neurologia

Rua Botucatu, 740 - Vila Clementino

04023-900 - São Paulo, SP

E-mail:newas@opus.com.br 